\title{
Pattern Formation of a Keller-Segel Model with the Source Term $u^{p}(1-u)$
}

\author{
Shengmao Fu ${ }^{1,2}$ and Fenli Cao ${ }^{1}$ \\ ${ }^{1}$ College of Mathematics and Statistics, Northwest Normal University, Lanzhou 730070, China \\ ${ }^{2}$ School of Mathematics and Computing Science, Guilin University of Electronic Technology, Guilin 541004, China
}

Correspondence should be addressed to Shengmao Fu; fusm@nwnu.edu.cn

Received 31 January 2013; Accepted 11 August 2013

Academic Editor: Liwei Zhang

Copyright (C) 2013 S. Fu and F. Cao. This is an open access article distributed under the Creative Commons Attribution License, which permits unrestricted use, distribution, and reproduction in any medium, provided the original work is properly cited.

Nonlinear dynamics near an unstable constant equilibrium in a Keller-Segel model with the source term $u^{p}(1-u)$ is considered. It is proved that nonlinear dynamics of a general perturbation is determined by the finite number of linear growing modes over a time scale of $\ln (1 / \delta)$, where $\delta$ is a strength of the initial perturbation.

\section{Introduction}

Mimura and Tsujikawa in [1] proposed a mathematical model for the pattern dynamics of aggregating regions of biological individuals possessing the property of chemotaxis, a dimensionless prototype of which reads

$$
\begin{gathered}
U_{t}=\nabla\left(D_{u} \nabla U-\chi U \nabla V\right)+g(U), \\
V_{t}=D_{v} \nabla^{2} V+\alpha U-\beta V,
\end{gathered}
$$

where $U(x, t)$ is the cell density, $V(x, t)$ is the concentration of chemotactic substance, $D_{u}>0$ is the amoeboid motility, $\chi>$ 0 is the chemotactic sensitivity, $D_{v}>0$ is the diffusion rate of cyclic adenosine monophosphate (cAMP), $\alpha>0$ is the rate of cAMP secretion per unit density of amoebae, and $\beta>0$ is the rate of degradation of cAMP in environment. In [1], the growth term $g(U)$ is classified into the three cases: (i) $g(0)=0$ and $g(u)<0$, for any $u>0$, (ii) (bistable type) $g(0)=g(a)=$ $g(1)=0$, for some $0<a<1, g(u)<0$, for $0<u<a$, and $g(u)>0$, for $a<u<1$, and (iii) (Logistic type) $g(0)=g(1)=$ 0 and $g(u)>0$, for $0<u<1$.

For model (1), with a Logistic source term $g(u)=u(1-u)$, Tello and Winkler [2] obtained infinitely many local branches of nonconstant stationary solutions bifurcating from a positive constant solution, while Kurata et al. [3] numerically showed several spatiotemporal patterns in a rectangle. Kuto et al. [4] considered some qualitative behaviors of stationary solutions from global and local (bifurcation) viewpoints.
Banerjee et al. [5] showed that the corresponding dynamics may lead to steady states, to divergences in a finite time as well as to the formation of spatiotemporal irregular patterns. Painter and Hillen [6] demonstrated the capacity of (1) to selforganize into multiple cellular aggregations, which, according to position in parameter space, either form a stationary pattern or undergo a sustained spatiotemporal sequence of merging (two aggregations coalesce) and emerging (a new aggregation appears). Numerical explorations into the latter indicate a positive Lyapunov exponent (sensitive dependence to initial conditions) together with a rich bifurcation structure. They argued that the spatiotemporal irregularity observed here describes a form of spatiotemporal chaos.

For model (1) with a logistic-like growth term $g(u)=$ $u^{2}(1-u)$, Aida et al. [7] estimated from below the attractor dimension of (1). Efendiev et al. [8] showed that dimension estimates of global attractors for the approximate systems are uniform with respect to the discretization parameter and polynomial order with respect to the chemotactic coefficient in the equation. By using nonnegativity of solutions, Nakaguchi and Efendiev [9] managed significantly to improve dimension estimates with respect to the chemotactic parameter. It is also well-known that the asymptotic behavior of solutions relating to patterns can be described by the dynamical systems of equations and that the degrees of freedom of such processes, which characterize the richness of emerging patterns, correspond to the dimensions of their attractors. 
Recently, Guo and Hwang in [10] investigated nonlinear dynamics near an unstable constant equilibrium in the classical Keller-Segel model (i.e., (1) with $g(u)=0$, see [11]). Their result can be interpreted as a rigorous mathematical characterization for pattern formation in the Keller-Segel model.

In the present paper, we consider the nonlinear dynamics near an unstable constant equilibrium for the following chemotaxis-diffusion-growth model:

$$
\begin{gathered}
U_{t}=\nabla(\nabla U-\chi U \nabla V)+U^{p}(1-U), \quad x \in \mathbb{T}^{d}, t>0, \\
V_{t}=\nabla^{2} V+U-\beta V, \quad x \in \mathbb{T}^{d}, t>0,
\end{gathered}
$$

which satisfies the homogeneous Neumann boundary conditions and initial value conditions for $U(x, t)$ and $V(x, t)$, that is,

$$
\begin{gathered}
\frac{\partial U}{\partial x_{i}}=\frac{\partial V}{\partial x_{i}}=0, \quad x_{i}=0, \pi, 1 \leq i \leq d, t>0, \\
U(x, 0)=U_{0}(x) \geq(\neq) 0, \\
V(x, 0)=V_{0}(x) \geq(\not) 0, \quad x \in \mathbb{T}^{d},
\end{gathered}
$$

where $\mathbb{T}^{d}=(0, \pi)^{d}(d=1,2,3)$ is a $d$-dimensional box, $p \geq$ 1. By using the bootstrap technique in [10] and higher-order energy estimates, we prove that given any general perturbation of magnitude $\delta$, its nonlinear evolution is dominated by the corresponding linear dynamics along a finite number of fixed fastest growing modes, over a time period of the order $\ln (1 / \delta)$. Each initial perturbation certainly can behave drastically differently from another, which gives rise to the richness of patterns.

\section{Local Stability of Positive Constant Equilibrium Solution}

The PDE system (2) without chemotaxis is as follows

$$
\begin{gathered}
U_{t}=\nabla^{2} U+U^{p}(1-U), \quad x \in \mathbb{T}^{d}(d=1,2,3), t>0, \\
V_{t}=\nabla^{2} V+U-\beta V, \quad x \in \mathbb{T}^{d}(d=1,2,3), t>0, \\
\frac{\partial U}{\partial x_{i}}=\frac{\partial V}{\partial x_{i}}=0, \quad \text { at } x_{i}=0, \pi, \text { for } 1 \leq i \leq d,
\end{gathered}
$$

$[\bar{U}, \bar{V}]=[1,1 / \beta]$ is the unique positive equilibrium point. We use $[\cdot, \cdot]$ to denote a column vector. Let $\mathbf{W}=[U, V], \overline{\mathbf{W}}=$ $[\bar{U}, \bar{V}]$, and $\mathbf{G}(\mathbf{W})=\left[U^{p}(1-U), U-\beta V\right]$. Then

$$
\mathbf{G}_{\mathbf{W}}(\overline{\mathbf{W}})=\left(\begin{array}{cc}
-1 & 0 \\
1 & -\beta
\end{array}\right) \text {. }
$$

Theorem 1. The positive equilibrium point $[\bar{U}, \bar{V}]$ of (5) is locally asymptotically stable.

Proof. Let $0=\mu_{1}<\mu_{2}<\mu_{3}<\cdots$ be the eigenvalues of the operator $-\Delta$ on $\mathbb{T}^{d}(d=1,2,3)$ with the homogeneous Neumann boundary condition and let $E\left(\mu_{i}\right)$ be the eigenspace corresponding to $\mu_{i}$ in $H^{1}\left(\mathbb{T}^{d}\right)$. Let $X=\left[H^{1}\left(\mathbb{T}^{d}\right)\right]^{2},\left\{\phi_{i j}: j=\right.$ $\left.1, \ldots, \operatorname{dim} E\left(\mu_{i}\right)\right\}$ be an orthonormal basis of $E\left(\mu_{i}\right)$ and $X_{i j}=$ $\left\{\mathbf{c} \cdot \phi_{i j} \mid \mathbf{c} \in \mathbb{R}^{2}\right\}$. Then

$$
X=\oplus_{i=1}^{\infty} X_{i}, \quad X_{i}=\oplus_{j=1}^{\operatorname{dim}\left[E\left(\mu_{i}\right)\right]} X_{i j} .
$$

Let $\mathfrak{D}=\operatorname{diag}(1,1)$ and $\mathfrak{L}=\mathfrak{D} \Delta+\mathbf{G}_{\mathbf{W}}(\overline{\mathbf{W}})$. The linearization of (5) at $[\bar{U}, \bar{V}]$ is

$$
\mathbf{W}_{t}=\mathfrak{Q}(\mathbf{W}-\overline{\mathbf{W}}) .
$$

For each $i \geq 1, X_{i}$ is invariant under the operator $\mathfrak{L}$, and $\lambda$ is an eigenvalue of $\mathfrak{L}$ on $X_{i}$, if and only if it is an eigenvalue of the matrix

$$
-\mu_{i} \mathfrak{D}+\mathbf{G}_{\mathbf{W}}(\overline{\mathbf{W}})=\left(\begin{array}{cc}
-\mu_{i}-1 & 0 \\
1 & -\mu_{i}-\beta
\end{array}\right) .
$$

Then $-\mu_{i} \mathfrak{D}+\mathbf{G}_{\mathbf{W}}(\overline{\mathbf{W}})$ has two negative eigenvalues $-\mu_{i}-1$ and $-\mu_{i}-\beta$. Hence, $[\bar{U}, \bar{V}]$ is locally asymptotically stable (see $[12])$.

\section{Growing Modes in the System (2)}

Let $u(\mathbf{x}, t)=U(\mathbf{x}, t)-\bar{U}, v(\mathbf{x}, t)=V(\mathbf{x}, t)-\bar{V}$. Then

$$
\begin{gathered}
u_{t}=\nabla^{2} u-\chi \nabla^{2} v-\chi \nabla(u \nabla v)-(u+1)^{p} u, \\
v_{t}=\nabla^{2} v+u-\beta v .
\end{gathered}
$$

The corresponding linearized system takes the form

$$
\begin{gathered}
u_{t}=\nabla^{2} u-\chi \nabla^{2} v-u, \\
v_{t}=\nabla^{2} v+u-\beta v .
\end{gathered}
$$

Let $\mathbf{w}(\mathbf{x}, t) \equiv[u(\mathbf{x}, t), v(\mathbf{x}, t)], \mathbf{q}=\left(q_{1}, \ldots, q_{d}\right) \in \Omega=\mathbb{N}^{d}$, and $e_{\mathbf{q}}(\mathbf{x})=\prod_{i=1}^{d} \cos \left(q_{i} x_{i}\right)$. Then $\left\{e_{\mathbf{q}}(\mathbf{x})\right\}_{\mathbf{q} \in \Omega}$ forms a basis of the space of functions in $\mathbb{T}^{d}$ that satisfy Neumann boundary conditions (3). To find a normal mode to the linear system (11) of the following form

$$
\mathbf{w}(\mathbf{x}, t)=\mathbf{r}_{\mathbf{q}} \exp \left(\lambda_{\mathbf{q}} t\right) e_{\mathbf{q}}(\mathbf{x}),
$$

where $\mathbf{r}_{\mathbf{q}}$ is a vector depending on $\mathbf{q}$, we substitute (12) into (11) to get

$$
\lambda_{\mathbf{q}} \mathbf{r}_{\mathbf{q}}=\left(\begin{array}{cc}
-q^{2}-1 & \chi q^{2} \\
1 & -q^{2}-\beta
\end{array}\right) \mathbf{r}_{\mathbf{q}},
$$

where $q^{2}=\sum_{i=1}^{d} q_{i}^{2}$. This implies that

$$
\begin{gathered}
\operatorname{det}\left(\begin{array}{cc}
\lambda_{\mathbf{q}}+q^{2}+1 & -\chi q^{2} \\
-1 & \lambda_{\mathbf{q}}+q^{2}+\beta
\end{array}\right)=0, \\
\lambda_{\mathbf{q}}^{2}+\left(2 q^{2}+\beta+1\right) \lambda_{\mathbf{q}}+\left(q^{2}+1\right)\left(q^{2}+\beta\right)-\chi q^{2}=0 .
\end{gathered}
$$

Thus, if there exists a $\mathbf{q}$ such that the linear instability criterion holds, that is,

$$
\left(q^{2}+1\right)\left(q^{2}+\beta\right)-\chi q^{2}<0,
$$


then (15) has at least one positive $\operatorname{root} \lambda_{\mathbf{q}}$. Obviously,

$$
\left(2 q^{2}+\beta+1\right)^{2}-4\left(q^{2}+1\right)\left(q^{2}+\beta\right)+4 \chi q^{2}>0 .
$$

Therefore, we can denote two distinct real roots for all $\mathbf{q}$ by

$$
\lambda_{\mathbf{q}}^{ \pm}=\frac{-\left(2 q^{2}+\beta+1\right) \pm \sqrt{(\beta-1)^{2}+4 \chi q^{2}}}{2} .
$$

The corresponding (linearly independent) eigenvectors $\mathbf{r}_{-}(\mathbf{q})$ and $\mathbf{r}_{+}(\mathbf{q})$ are given by

$$
\mathbf{r}_{ \pm}(\mathbf{q})=\left[\lambda_{\mathbf{q}}^{ \pm}+q^{2}+\beta, 1\right] .
$$

It is easy to see from (16) that there exist only finitely many $q$, such that $\lambda_{\mathbf{q}}^{+}>0$. We therefore denote the largest eigenvalue by $\lambda_{\max }>0$ and define

$$
\Omega_{\max } \equiv\left\{\mathbf{q} \in \Omega: \lambda_{\mathbf{q}}^{+}=\lambda_{\max }\right\}
$$

Moreover, there is one $q^{2}$ (possibly two) having $\lambda^{+}\left(q^{2}\right)=$ $\lambda_{\text {max }}$, when we regard $\lambda_{\mathbf{q}}^{+}$as a function of $q^{2}$. We also denote $\nu>0$ to be the gap between the $\lambda_{\max }$ and the rest, that is,

$$
\nu=\min _{\mathbf{q} \in \Omega \backslash \Omega_{\max }}\left|\lambda_{\max }-\lambda_{\mathbf{q}}\right|
$$

Given any initial perturbation $\mathbf{w}(\mathbf{x}, 0)$, that is,

$$
\mathbf{w}(\mathbf{x}, 0)=\sum_{\mathbf{q} \in \Omega} \mathbf{w}_{\mathbf{q}} e_{\mathbf{q}}(\mathbf{x})=\sum_{\mathbf{q} \in \Omega}\left\{w_{\mathbf{q}}^{-} \mathbf{r}_{-}(\mathbf{q})+w_{\mathbf{q}}^{+} \mathbf{r}_{+}(\mathbf{q})\right\} e_{\mathbf{q}}(\mathbf{x}),
$$

where

$$
\mathbf{w}_{\mathbf{q}}=w_{\mathbf{q}}^{-} \mathbf{r}_{-}(\mathbf{q})+w_{\mathbf{q}}^{+} \mathbf{r}_{+}(\mathbf{q}),
$$

we know that the unique solution $\mathbf{w}(\mathbf{x}, t)=[u(\mathbf{x}, t), v(\mathbf{x}, t)]$ of (11) is given by

$$
\begin{gathered}
\mathbf{w}(\mathbf{x}, t)=\sum_{\mathbf{q} \in \Omega}\left\{w_{\mathbf{q}}^{-} \mathbf{r}_{-}(\mathbf{q}) \exp \left(\lambda_{\mathbf{q}}^{-} t\right)+w_{\mathbf{q}}^{+} \mathbf{r}_{+}(\mathbf{q}) \exp \left(\lambda_{\mathbf{q}}^{+} t\right)\right\} \\
\times e_{\mathbf{q}}(\mathbf{x}) \equiv e^{\mathfrak{\Omega} t} \mathbf{w}(\mathbf{x}, 0) .
\end{gathered}
$$

For any $\mathbf{g}(\cdot, t) \in\left[L^{2}\left(\mathbb{T}^{d}\right)\right]^{2}$, we denote $\|\mathbf{g}(\cdot, t)\| \equiv\|\mathbf{g}(\cdot, t)\|_{L^{2}}$ and $\langle\cdot, \cdot\rangle$ and $(\cdot, \cdot)$ are the inner product of $\left[L^{2}\left(\mathbb{T}^{d}\right)\right]^{2}$ and the scaler product of $\mathbb{R}^{2}$, respectively. Our main result of this section is the following lemma.

Lemma 2. Assume that the instability criterion (16) holds. Let

$$
\mathbf{w}(\mathbf{x}, t)=[u(\mathbf{x}, t), v(\mathbf{x}, t)] \equiv e^{\mathfrak{\Omega} t} \mathbf{w}(\mathbf{x}, 0)
$$

be a solution to the linearized system (11) with initial condition $\mathbf{w}(\mathbf{x}, 0)$. Then there exists a constant $C_{1} \geq 1$ depending on $\chi$ and $\beta$, such that

$$
\|\mathbf{w}(\cdot, t)\| \leq C_{1} \exp \left(\lambda_{\max } t\right)\|\mathbf{w}(\cdot, 0)\|, \quad \forall t>0 .
$$

Proof. We prove the Lemma in the following two cases.

(1) $t \geq 1$. By analyzing (15), we have

$$
\lim _{\mathbf{q} \rightarrow \infty} \frac{\lambda_{\mathbf{q}}^{ \pm}}{q^{2}}=-1
$$

It follows from (18) that

$$
\frac{\lambda_{\mathbf{q}}^{+}-\lambda_{\mathbf{q}}^{-}}{q^{2}} \geq \frac{2 \sqrt{\chi}}{q} .
$$

By solving (23), we have

$$
\left|w_{\mathbf{q}}^{ \pm}\right| \leq \frac{\left|\mathbf{r}_{ \pm}(\mathbf{q})\right| \times\left|\mathbf{w}_{\mathbf{q}}\right|}{\left|\operatorname{det}\left[\mathbf{r}_{-}(\mathbf{q}), \mathbf{r}_{+}(\mathbf{q})\right]\right|}
$$

Clearly,

$$
\left|\mathbf{r}_{ \pm}(\mathbf{q})\right|=\sqrt{\left(\frac{\lambda_{\mathbf{q}}^{ \pm}}{q^{2}} \cdot q^{2}+q^{2}+\beta\right)^{2}+1}
$$

Later on, we will always denote universal constants by $E_{i}(i=1,2, \ldots)$. It is not hard to verify that there exist positive constants $E_{1}$ and $E_{2}$, such that

$$
\left|\lambda_{\mathbf{q}}^{ \pm}\right| \leq E_{1} q^{2}, \quad\left|\mathbf{r}_{ \pm}(\mathbf{q})\right| \leq 2 E_{2} q^{2}
$$

where $E_{2}=\max \left\{E_{1}+1, \beta+1\right\}$. It follows from (19) and (28) that

$$
\frac{1}{\left|\operatorname{det}\left[\mathbf{r}_{-}(\mathbf{q}), \mathbf{r}_{+}(\mathbf{q})\right]\right|} \leq \frac{1}{2 q} \sqrt{\frac{1}{\chi}}
$$

Combining (29)-(32), we can obtain

$$
\left|w_{\mathbf{q}}^{ \pm}\right| \leq E_{3} q\left|\mathbf{w}_{\mathbf{q}}\right|
$$

where $E_{3}=E_{2} \sqrt{1 / \chi}$. From (31) and (33), we have

$$
\left|w_{\mathbf{q}}^{ \pm} \mathbf{r}_{ \pm}(\mathbf{q}) \exp \left(\lambda_{\mathbf{q}}^{ \pm} t\right)\right| \leq 2 E_{2} E_{3} q^{3}\left|\mathbf{w}_{\mathbf{q}}\right| \exp \left(\lambda_{\mathbf{q}}^{ \pm} t\right) .
$$

For $t \geq 1$, it follows from (18) that

$$
\begin{aligned}
\exp \left(\lambda_{\mathbf{q}}^{ \pm} t\right) & \leq \exp \left\{-\frac{q^{2}\left(q^{2}+\beta+1-\chi\right)+\beta}{2 q^{2}+\beta+1}\right\} \\
& \leq \exp \left\{-\frac{q^{2}+\beta+1-\chi}{\beta+3}\right\} .
\end{aligned}
$$

Therefore, for all $q>0$, there exists a constant $E_{4}>0$, such that

$$
q^{3} \exp \left(\lambda_{\mathbf{q}}^{ \pm} t\right) \leq E_{4}
$$

From (34) and (36), we obtain

$$
\left|w_{\mathbf{q}}^{ \pm} \mathbf{r}_{ \pm}(\mathbf{q}) \exp \left(\lambda_{\mathbf{q}}^{ \pm} t\right)\right| \leq E_{5}\left|\mathbf{w}_{\mathbf{q}}\right|,
$$


where $E_{5}=2 E_{2} E_{3} E_{4}$. By (23), we have

$$
\begin{aligned}
\left|\mathbf{w}_{\mathbf{q}}\right|^{2}= & w_{\mathbf{q}}^{-2}\left|\mathbf{r}_{-}(\mathbf{q})\right|^{2} \\
& +2 w_{\mathbf{q}}^{-} w_{\mathbf{q}}^{+}\left(\mathbf{r}_{-}(\mathbf{q}), \mathbf{r}_{+}(\mathbf{q})\right)+w_{\mathbf{q}}^{+2}\left|\mathbf{r}_{+}(\mathbf{q})\right|^{2} .
\end{aligned}
$$

Notice that

$$
\|\mathbf{w}(\mathbf{x}, 0)\|^{2}=\left(\frac{\pi}{2}\right)^{d} \sum_{\mathbf{q} \in \Omega}\left|\mathbf{w}_{\mathbf{q}}\right|^{2}
$$

It follows from (37) and (39) that

$$
\begin{aligned}
\|\mathbf{w}(\mathbf{x}, t)\| \leq & \left\|\sum_{\mathbf{q} \in \Omega} w_{\mathbf{q}}^{-} \mathbf{r}_{-}(\mathbf{q}) \exp \left(\lambda_{\mathbf{q}}^{-} t\right) e_{\mathbf{q}}(\mathbf{x})\right\| \\
& +\left\|\sum_{\mathbf{q} \in \Omega} w_{\mathbf{q}}^{+} \mathbf{r}_{+}(\mathbf{q}) \exp \left(\lambda_{\mathbf{q}}^{+} t\right) e_{\mathbf{q}}(\mathbf{x})\right\| \\
\leq & \left(\left(\frac{\pi}{2}\right)^{d} \sum_{\mathbf{q} \in \Omega} E_{5}^{2}\left|\mathbf{w}_{\mathbf{q}}\right|^{2}\right)^{1 / 2} \\
& +\left(\left(\frac{\pi}{2}\right)^{d} \sum_{\mathbf{q} \in \Omega} E_{5}^{2}\left|\mathbf{w}_{\mathbf{q}}\right|^{2}\right)^{1 / 2} \\
= & 2 E_{5}\|\mathbf{w}(\mathbf{x}, 0)\| .
\end{aligned}
$$

Therefore,

$$
\|\mathbf{w}(\mathbf{x}, t)\| \leq 2 E_{5} \exp \left(\lambda_{\max } t\right)\|\mathbf{w}(\mathbf{x}, 0)\|, \quad \forall t \geq 1 .
$$

(2) $t \leq 1$. By (11), we have

$$
\begin{aligned}
& \frac{1}{2} \frac{d}{d t} \int_{\mathbb{T}^{d}}\left\{|u|^{2}+A|v|^{2}\right\} \mathbf{d} \mathbf{x} \\
& \quad+\int_{\mathbb{T}^{d}}\left\{|\nabla u|^{2}+A|\nabla v|^{2}-\chi \nabla u \nabla v\right\} \mathbf{d x} \mathbf{x} \\
& \quad+\int_{\mathbb{T}^{d}} u^{2} \mathbf{d} \mathbf{x}+A \beta \int_{\mathbb{T}^{d}} v^{2} \mathbf{d} \mathbf{x}=A \int_{\mathbb{T}^{d}} u v \mathbf{d} \mathbf{x} .
\end{aligned}
$$

If $A=\chi^{2}$, then the integrand of the second integral can be chosen nonnegative as follows:

$$
|\nabla u|^{2}+A|\nabla v|^{2}-\chi \nabla u \nabla v \geq \frac{1}{2}|\nabla u|^{2}+\frac{\chi^{2}}{2}|\nabla v|^{2} \geq 0 .
$$

Combining (42) and Young inequality together, we derive

$$
\begin{aligned}
& \frac{1}{2} \frac{d}{d t} \int_{\mathbb{T}^{d}}\left\{|u|^{2}+A|v|^{2}\right\} \mathbf{d} \mathbf{x} \\
& \leq \frac{A}{2} \int_{\mathbb{T}^{d}}\left\{|u|^{2}+|v|^{2}\right\} \mathbf{d} \mathbf{x} .
\end{aligned}
$$

By Grownwall inequality, we can obtain

$$
\|\mathbf{w}(\mathbf{x}, t)\| \leq C_{1} \exp \left(\lambda_{\max } t\right)\|\mathbf{w}(\mathbf{x}, 0)\|,
$$

where $C_{1}=\max \left\{2 E_{5},((\exp A) / A)^{1 / 2}\right\} \geq 1$, if $0<A<1$; $C_{1}=\max \left\{2 E_{5},(A \exp A)^{1 / 2}\right\} \geq 1$, if $A>1$.

\section{Bootstrap Lemma}

By a standard PDE theory [13], one can establish the existence of local solutions for (10).
Lemma 3 (local existence). For $s \geq 1(d=1)$ and $s \geq 2(d=$ $2,3)$, there exists a $T_{0}>0$, such that $(10)$ with $u(\cdot, 0), v(\cdot, 0) \in$ $H^{s}$ has a unique solution $\mathbf{w}(\cdot, t)$ on $\left(0, T_{0}\right)$, which satisfies

$$
\|\mathbf{w}(t)\|_{H^{s}} \leq C\|\mathbf{w}(0)\|_{H^{s}}, \quad 0<t<T_{0},
$$

where $C$ is a positive constant depending on $\chi$ and $\beta$.

Lemma 4. Let $[u(\mathbf{x}, t), v(\mathbf{x}, t)]$ be a solution of $(10)$. Then

$$
\begin{aligned}
\frac{1}{2} \frac{d}{d t} \sum_{|\alpha|=2} \int_{2 \mathbb{J}^{d}}\left\{\left|D^{\alpha} u\right|^{2}+\chi^{2}\left|D^{\alpha} v\right|^{2}\right\} \mathbf{d} \mathbf{x} \\
\quad+\sum_{|\alpha|=2} \int_{2 \mathbb{J}^{d}}\left\{\frac{1}{4}\left|\nabla D^{\alpha} u\right|^{2}+\frac{\chi^{2}}{2}\left|\nabla D^{\alpha} v\right|^{2}\right\} \mathbf{d} \mathbf{x} \\
\quad+\frac{A \beta}{2} \sum_{|\alpha|=2} \int_{2 \mathbb{J}^{d}}\left|D^{\alpha} v\right|^{2} \mathbf{d} \mathbf{x} \\
\leq C \\
\quad \times\left(\chi+2^{p-1}(p+1)^{2}\right\} \\
\times\left(\|\mathbf{w}\|_{H^{2}}+\|\mathbf{w}\|_{H^{2}}^{p}\right)\left\|\nabla^{3} \mathbf{w}\right\|^{2}+C^{\prime}\|u\|^{2},
\end{aligned}
$$

where $C$ is the universal constant and $C^{\prime}=4\{A / 2 \beta-1\}^{3}$.

Proof. Notice that if $\mathbf{w}(x, t)$ is a solution of $(10)$ on $(0, \pi)$, then $\mathbf{w}\left(-x_{i}, t\right)$ is a solution of $(10)$ on $(-\pi, 0)$. Let $[\widetilde{u}(\mathbf{x}, t), \widetilde{v}(\mathbf{x}, t)]$ be a solution of $(10)$ on $(-\pi, \pi)^{d}$, which is an even extension of $[u(\mathbf{x}, t), v(\mathbf{x}, t)]$ on $(-\pi, 0)^{d}$. Then one may assume periodicity at the boundary of the extended $2 \mathbb{T}^{d} \equiv(-\pi, \pi)^{d}$.

By (10), one has

$$
\begin{aligned}
& \frac{1}{2} \frac{d}{d t} \int_{2 \mathbb{J}^{d}}\left\{\left|\partial_{x_{i} x_{j}} \widetilde{u}\right|^{2} \mathbf{d x}+A\left|\partial_{x_{i} x_{j}} \widetilde{v}\right|^{2} \mathbf{d x}\right\} \\
& =-\int_{2 \mathbb{J}^{d}}\left\{\left|\nabla \partial_{x_{i} x_{j}} \tilde{u}\right|^{2}+A\left|\nabla \partial_{x_{i} x_{j}} \widetilde{v}\right|^{2}\right. \\
& \left.-\chi \nabla \partial_{x_{i} x_{j}} \widetilde{u} \nabla \partial_{x_{i} x_{j}} \widetilde{v}\right\} \mathbf{d x} \\
& -\int_{2 \mathbb{J}^{d}}\left|\partial_{x_{i} x_{j}} \widetilde{u}\right|^{2} \mathbf{d x} \\
& +A \int_{2 \mathbb{\Psi}^{d}} \partial_{x_{i} x_{j}} \widetilde{u} \partial_{x_{i} x_{j}} \widetilde{v} \mathbf{d x} \\
& -A \beta \int_{2 \mathbb{T}^{d}}\left|\partial_{x_{i} x_{j}} \widetilde{v}\right|^{2} \mathbf{d} \mathbf{x} \\
& +\chi \int_{2 \mathbb{J}^{d}} \nabla \partial_{x_{i} x_{j}} \tilde{u} \cdot \partial_{x_{i} x_{j}}\{\tilde{u} \nabla \tilde{v}\} \mathbf{d} \mathbf{x} \\
& +\int_{2 \mathbb{\Psi}^{d}}\left\{\left|\partial_{x_{i} x_{j}} \tilde{u}\right|^{2}\right. \\
& \left.-\partial_{x_{i} x_{j}} \tilde{u} \partial_{x_{i} x_{j}}\left[v \widetilde{u}(\widetilde{u}+1)^{p}\right]\right\} \mathbf{d} \mathbf{x} \\
& =: I_{1}+I_{2} \text {, }
\end{aligned}
$$


where $A=\chi^{2}$, and

$$
\begin{aligned}
I_{1}= & -\int_{2 \mathbb{T}^{d}}\left\{\left|\nabla \partial_{x_{i} x_{j}} \widetilde{u}\right|^{2}+A\left|\nabla \partial_{x_{i} x_{j}} \widetilde{v}\right|^{2}\right. \\
& \left.-\chi \nabla \partial_{x_{i} x_{j}} \widetilde{u} \nabla \partial_{x_{i} x_{j}} \widetilde{v}\right\} \mathbf{d} \mathbf{x} \\
& -A \beta \int_{2 \mathbb{J}^{d}}\left|\partial_{x_{i} x_{j}} \widetilde{v}\right|^{2} \mathbf{d} \mathbf{x} \\
& +A \int_{2 \mathbb{T}^{d}} \partial_{x_{i} x_{j}} \widetilde{u} \partial_{x_{i} x_{j}} \widetilde{v} \mathbf{d} \mathbf{x} \\
& -\int_{2 \mathbb{T}^{d}}\left|\partial_{x_{i} x_{j}} \widetilde{u}\right|^{2} \mathbf{d} \mathbf{x} .
\end{aligned}
$$

Clearly,

$$
\begin{gathered}
\left|\nabla \partial_{x_{i} x_{j}} \widetilde{u}\right|^{2}+A\left|\nabla \partial_{x_{i} x_{j}} \widetilde{v}\right|^{2}-\chi \nabla \partial_{x_{i} x_{j}} \widetilde{u} \cdot \nabla \partial_{x_{i} x_{j}} \widetilde{v} \\
\geq \frac{1}{2}\left|\nabla \partial_{x_{i} x_{j}} \widetilde{u}\right|^{2}+\frac{\chi^{2}}{2}\left|\nabla \partial_{x_{i} x_{j}} \widetilde{v}\right|^{2} .
\end{gathered}
$$

Thus,

$$
\begin{aligned}
I_{1} \leq & -\int_{2 \mathbb{T}^{d}}\left\{\frac{1}{2}\left|\nabla \partial_{x_{i} x_{j}} \widetilde{u}\right|^{2}+\frac{\chi^{2}}{2}\left|\nabla \partial_{x_{i} x_{j}} \widetilde{v}\right|^{2}\right\} \mathbf{d} \mathbf{x} \\
& -\frac{A \beta}{2} \int_{2 \mathbb{T}^{d}}\left|\partial_{x_{i} x_{j}} \widetilde{v}\right|^{2} \mathbf{d x} \\
& +\left(\frac{A}{2 \beta}-1\right) \int_{2 \mathbb{T}^{d}}\left|\partial_{x_{i} x_{j}} \widetilde{u}\right|^{2} \mathbf{d x} .
\end{aligned}
$$

By interpolation inequalities, one knows that

$$
\left\|\partial_{x_{i} x_{j}} \tilde{u}\right\|^{2} \leq a\left\|\nabla \partial_{x_{i} x_{j}} \widetilde{u}\right\|^{2}+\frac{1}{4 a^{2}}\|\widetilde{u}\|^{2},
$$

for any $a>0$. If $A \leq 2 \beta$, then one can delete the last term in the above inequality. If $A>2 \beta$, one can choose positive constant $a$ such that $\{A / 2 \beta-1\} a=1 / 4$. Then

$$
\begin{aligned}
I_{1} \leq & -\int_{2 \mathbb{T}^{d}}\left\{\frac{1}{4}\left|\nabla \partial_{x_{i} x_{j}} \tilde{u}\right|^{2}\right. \\
& \left.+\frac{\chi^{2}}{2}\left|\nabla \partial_{x_{i} x_{j}} \widetilde{v}\right|^{2}\right\} \mathbf{d} \mathbf{x} \\
& -\frac{A \beta}{2} \int_{2 \mathbb{J}^{d}}\left|\partial_{x_{i} x_{j}} \widetilde{v}\right|^{2} \mathbf{d x}+C^{\prime}\|\widetilde{u}\|^{2},
\end{aligned}
$$

where $C^{\prime}=4\{A / 2 \beta-1\}^{3}$.

One now estimates the second nonlinear term $I_{2}$ :

$$
\begin{aligned}
I_{2}= & \chi \int_{2 \mathbb{J}^{d}} \nabla \partial_{x_{i} x_{j}} \tilde{u} \cdot \partial_{x_{i} x_{j}}\{\widetilde{u} \nabla \widetilde{v}\} \mathbf{d x} \\
& +\int_{2 \mathbb{J}^{d}}\left\{\left|\partial_{x_{i} x_{j}} \widetilde{u}\right|^{2}-\partial_{x_{i} x_{j}} \widetilde{u} \partial_{x_{i} x_{j}}\right. \\
& \left.\times\left[\widetilde{u}(\widetilde{\mathcal{u}}+1)^{p}\right]\right\} \mathbf{d x} \\
= & I_{2}^{\prime}+I_{2}^{\prime \prime} .
\end{aligned}
$$

Clearly,

$$
\begin{aligned}
I_{2}^{\prime} \leq \chi\{ & \left\{\nabla \widetilde{v}\left\|_{L^{\infty}}\right\| \nabla \partial_{x_{i} x_{j}} \widetilde{u}\|\| \partial_{x_{i} x_{j}} \widetilde{u} \|\right. \\
& +2 \sum_{i=1}^{d}\|\nabla \widetilde{u}\|_{L^{\infty}}\left\|\partial_{x_{i} x_{j}} \widetilde{v}\right\|\left\|\nabla \partial_{x_{i} x_{j}} \widetilde{u}\right\| \\
& \left.+\|\widetilde{u}\|_{L^{\infty}}\left\|\nabla \partial_{x_{i} x_{j}} \widetilde{u}\right\|\left\|\nabla \partial_{x_{i} x_{j}} \widetilde{v}\right\|\right\} .
\end{aligned}
$$

Notice that

$$
\|g\|_{L^{\infty}\left(2 \mathbb{T}^{d}\right)} \leq E_{0}\|g\|_{H^{2}\left(2 \mathbb{T}^{d}\right)}, \quad \text { for } d \leq 3,
$$

$$
\begin{gathered}
\int_{2 \mathbb{T}^{d}} \nabla \widetilde{u} \mathbf{d} \mathbf{x}=\int_{2 \mathbb{J}^{d}} \nabla \widetilde{v} \mathbf{d} \mathbf{x}=0, \\
\int_{2 \mathbb{J}^{d}} \partial_{x_{i} x_{j}} \widetilde{u} \mathbf{d} \mathbf{x}=\int_{2 \mathbb{J}^{d}} \partial_{x_{i} x_{j}} \widetilde{v} \mathbf{d} \mathbf{x}=0, \\
\|g\| \leq\|g\|_{L^{6}\left(2 \mathbb{T}^{d}\right)} \\
\leq E_{1}\|g\|_{H^{1}} \leq E_{1}\|\nabla g\| \quad \text { if } d \leq 3,
\end{gathered}
$$

$$
\|\nabla g\|_{H^{2}} \leq E_{2}\left(\sum_{|\alpha|=2}\left\|\nabla D^{\alpha} g\right\|^{2}\right)^{1 / 2} \text {. }
$$

From (56)-(59), one can obtain

$$
\begin{aligned}
& I_{2}^{\prime} \leq \chi\left\{E_{0}\|\widetilde{u}\|_{H^{2}}\|\nabla \widetilde{v}\|_{H^{2}}\left\|\nabla \partial_{x_{i} x_{j}} \widetilde{u}\right\|\right. \\
& +2 d E_{0}\|\widetilde{v}\|_{H^{2}}\|\nabla \widetilde{u}\|_{H^{2}}\left\|\nabla \partial_{x_{i} x_{j}} \widetilde{u}\right\| \\
& \left.+E_{0}\|\widetilde{u}\|_{H^{2}}\left\|\nabla \partial_{x_{i} x_{j}} \widetilde{u}\right\|\left\|\nabla \partial_{x_{i} x_{j}} \widetilde{v}\right\|\right\} \\
& \leq \chi\left(E_{0} E_{2}+2 d E_{0} E_{2}+E_{0}\right) \\
& \times\|\widetilde{\mathbf{w}}\|_{H^{2}} \sum_{|\alpha|=2}\left\|\nabla D^{\alpha} \widetilde{\mathbf{w}}\right\|^{2} \\
& \leq \chi E_{3}\|\widetilde{\mathbf{w}}\|_{H^{2}}\left\|\nabla^{3} \widetilde{\mathbf{w}}\right\|^{2} .
\end{aligned}
$$

Similarly, one has

$$
\begin{aligned}
I_{2}^{\prime \prime} \leq & \int_{2 \mathbb{J}^{d}}\left\{\left|\partial_{x_{i} x_{j}} \widetilde{u}\right|^{2}-(\widetilde{u}+1)^{p}\left|\partial_{x_{i} x_{j}} \widetilde{u}\right|^{2}\right\} \mathbf{d} \mathbf{x} \\
& +p|p-1| \int_{2 \mathbb{T}^{d}}\left|(\widetilde{u}+1)^{p-2} \widetilde{u} \partial_{x_{i}} \widetilde{u} \partial_{x_{j}} \widetilde{u} \partial_{x_{i} x_{j}} \widetilde{u}\right| \mathbf{d} \mathbf{x} \\
& +\left.p \int_{2 \mathbb{T}^{d}}\left|(\widetilde{u}+1)^{p-1} \widetilde{u}\right| \partial_{x_{i} x_{j}} \widetilde{u}\right|^{2} \mid \mathbf{d x} \\
& +2 p \int_{2 \mathbb{J}^{d}}\left|(\widetilde{u}+1)^{p-1} \partial_{x_{i}} \widetilde{u} \partial_{x_{j}} \widetilde{u} \partial_{x_{i} x_{j}} \widetilde{u}\right| \mathbf{d x} \\
\leq & 2^{p-1} M_{2} E_{0}^{p}\left\{p(p+1) E_{2}^{2}\right. \\
& \left.+\left(p+M_{1}\right) E_{1}^{2}\right\}\|\widetilde{\mathbf{w}}\|_{H^{2}}^{p}\left\|\nabla^{3} \widetilde{\mathbf{w}}\right\|^{2} .
\end{aligned}
$$


Here and later, $M_{1}, M_{2}, \ldots$ are universal constants. It follows from (60) and (61) that

$$
\begin{aligned}
I_{2} \leq & \chi E_{3}\|\widetilde{\mathbf{w}}\|_{H^{2}}\left\|\nabla^{3} \widetilde{\mathbf{w}}\right\|^{2} \\
& +2^{p-1} M_{2} E_{0}^{p}\left\{p(p+1) E_{2}^{2}+\left(p+M_{1}\right) E_{1}^{2}\right\} \\
& \times\|\widetilde{\mathbf{w}}\|_{H^{2}}^{p}\left\|\nabla^{3} \widetilde{\mathbf{w}}\right\|^{2} \\
\leq & C\left\{\chi+2^{p-1}(p+1)^{2}\right\} \\
& \times\left(\|\widetilde{\mathbf{w}}\|_{H^{2}}+\|\widetilde{\mathbf{w}}\|_{H^{2}}^{p}\right)\left\|\nabla^{3} \widetilde{\mathbf{w}}\right\|^{2},
\end{aligned}
$$

where $C=\max \left\{E_{3}, M_{2} E_{0}^{p} E_{2}^{2}, M_{2} E_{0}^{p} E_{1}^{2}, M_{1} M_{2} E_{0}^{p} E_{1}^{2}\right\}$.

Recall that $[\widetilde{u}, \widetilde{v}]$ is the even extension of $[u, v]$. Combining (49), (53), and (62), one has

$$
\begin{aligned}
\frac{1}{2} \frac{d}{d t} \sum_{|\alpha|=2} \int_{2 \mathbb{J}^{d}}\left\{\left|D^{\alpha} u\right|^{2}+\chi^{2}\left|D^{\alpha} v\right|^{2}\right\} \mathbf{d x} \\
\quad+\sum_{|\alpha|=2} \int_{2 \mathbb{J}^{d}}\left\{\frac{1}{4}\left|\nabla D^{\alpha} u\right|^{2}+\frac{\chi^{2}}{2}\left|\nabla D^{\alpha} v\right|^{2}\right\} \mathbf{d} \mathbf{x} \\
\quad+\frac{A \beta}{2} \sum_{|\alpha|=2} \int_{2 \mathbb{J}^{d}}\left|D^{\alpha} v\right|^{2} \mathbf{d} \mathbf{x} \\
\leq C \\
\quad\left\{\chi+2^{p-1}(p+1)^{2}\right\} \\
\times\left(\|\mathbf{w}\|_{H^{2}}+\|\mathbf{w}\|_{H^{2}}^{p}\right)\left\|\nabla^{3} \mathbf{w}\right\|^{2}+C^{\prime}\|u\|^{2},
\end{aligned}
$$

where $C=\max \left\{E_{0}^{p}\left(E_{1}^{2}+E_{2}^{2}\right)+E_{1}^{2}, E_{3}\right\}$ and $C^{\prime}=4\{A / 2 \beta-$ $1\}^{3}$.

Lemma 5. Let $\mathbf{w}(\mathbf{x}, t)$ be a solution of (10), such that for $0 \leq$ $t \leq T<T_{0}$,

$$
\begin{gathered}
\|\mathbf{w}(\cdot, t)\|_{H^{2}}+\|\mathbf{w}(\cdot, t)\|_{H^{2}}^{p} \\
\leq \frac{1}{C} \min \left\{\frac{1}{4\left(\chi+2^{p-1}(p+1)^{2}\right)},\right. \\
\left.\frac{\chi^{2}}{2\left(\chi+2^{p-1}(p+1)^{2}\right)}\right\}, \\
\|\mathbf{w}(\cdot, t)\| \leq 2 C_{1} \exp \left(\lambda_{\max } t\right)\|\mathbf{w}(\cdot, 0)\| .
\end{gathered}
$$

Then

$$
\begin{array}{r}
\|\mathbf{w}(\cdot, t)\|_{H^{2}}^{p} \leq C_{3}\left\{\|\mathbf{w}(\cdot, 0)\|_{H^{2}}^{2}+\exp \left(2 \lambda_{\max } t\right)\|\mathbf{w}(\cdot, 0)\|^{2}\right\}^{p / 2}, \\
0 \leq t \leq T,
\end{array}
$$

where $C_{3}=\max \left\{\left(\left(E_{1}^{2}+1\right) \chi^{2}\right)^{p / 2},\left\{4 C_{1}^{2}\left[1+\left(E_{1}^{2}+1\right) C^{\prime} / \lambda_{\max }\right]\right\}^{p / 2}\right\}$, if $\chi^{2} \geq 1$ and $C_{3}=\max \left\{\left(\left(E_{1}^{2}+1\right) / \chi^{2}\right)^{p / 2},\left\{4 C_{1}^{2}\left[1+\left(E_{1}^{2}+\right.\right.\right.\right.$ 1) $\left.\left.\left.C^{\prime} / \lambda_{\max } \chi^{2}\right]\right\}^{p / 2}\right\}$, if $\chi^{2}<1$.
Proof. By (58), we have

$$
\|\nabla \widetilde{\mathbf{w}}(\cdot, t)\|^{2} \leq E_{1}^{2} \sum_{|\alpha|=2}\left\|D^{\alpha} \widetilde{\mathbf{w}}(\cdot, t)\right\|^{2} .
$$

It follows from (67) that

$$
\begin{aligned}
& \|\mathbf{w}(\cdot, t)\|_{H^{2}}^{p} \\
& \quad \leq\left(\|\mathbf{w}(\cdot, t)\|^{2}+\left(E_{1}^{2}+1\right) \sum_{|\alpha|=2}\left\|D^{\alpha} \mathbf{w}(\cdot, t)\right\|^{2}\right)^{p / 2} .
\end{aligned}
$$

Now, we estimate the second-order derivatives of $\mathbf{w}(\cdot, t)$. By Lemma 4, we have

$$
\begin{gathered}
\frac{1}{2} \frac{d}{d t} \sum_{|\alpha|=2} \int_{\mathbb{T}^{d}}\left\{\left|D^{\alpha} u\right|^{2}+\chi^{2}\left|D^{\alpha} v\right|^{2}\right\} \mathbf{d x} \mathbf{x} \\
\leq C^{\prime}\|u\|^{2} \leq C^{\prime}\|\mathbf{w}(\cdot, t)\|^{2} .
\end{gathered}
$$

Integrating on both sides of (69) from 0 to $t$, we get from (65) the following:

$$
\begin{gathered}
\sum_{|\alpha|=2} \int_{\mathbb{T}^{d}}\left\{\left|D^{\alpha} u(\cdot, t)\right|^{2}+\chi^{2}\left|D^{\alpha} v(\mathbf{x}, t)\right|^{2}\right\} \mathbf{d x} \\
\leq \sum_{|\alpha|=2} \int_{\mathbb{T}^{d}}\left\{\left|D^{\alpha} u(\cdot, 0)\right|^{2}\right. \\
\left.+\chi^{2}\left|D^{\alpha} v(\cdot, 0)\right|^{2}\right\} \mathbf{d x} \\
+\frac{4 C_{1}^{2} C^{\prime}}{\lambda_{\max }}\|\mathbf{w}(\cdot, 0)\|^{2} \exp \left(2 \lambda_{\max } t\right) .
\end{gathered}
$$

We will proceed in the following two cases: $\chi^{2} \geq 1$ and $\chi^{2}<1$.

(1) If $\chi^{2} \geq 1$, we have

$$
\begin{aligned}
\sum_{|\alpha|=2}\left\|D^{\alpha} \mathbf{w}(\cdot, t)\right\|^{2} & \\
\leq & \sum_{|\alpha|=2} \int_{\mathbb{J}^{d}}\left\{\left|D^{\alpha} u(\cdot, 0)\right|^{2}+\chi^{2}\left|D^{\alpha} v(\cdot, 0)\right|^{2}\right\} \mathbf{d x} \\
& +\frac{4 C_{1}^{2} C^{\prime}}{\lambda_{\max }}\|\mathbf{w}(\cdot, 0)\|^{2} \exp \left(2 \lambda_{\max } t\right) \\
\leq & \chi^{2} \sum_{|\alpha|=2}\left\|D^{\alpha} \mathbf{w}(\cdot, 0)\right\|^{2} \\
& +\frac{4 C_{1}^{2} C^{\prime}}{\lambda_{\max }}\|\mathbf{w}(\cdot, 0)\|^{2} \exp \left(2 \lambda_{\max } t\right) .
\end{aligned}
$$

By (68) and (71), one can obtain

$$
\begin{aligned}
\|\mathbf{w}(\cdot, t)\|_{H^{2}}^{p} \leq\{ & \left(E_{1}^{2}+1\right) \chi^{2} \sum_{|\alpha|=2}\left\|D^{\alpha} \mathbf{w}(\cdot, 0)\right\|^{2} \\
& +\left\{4 C_{1}^{2}+\left(E_{1}^{2}+1\right) \frac{4 C_{1}^{2} C^{\prime}}{\lambda_{\max }}\right\} \\
& \left.\times\|\mathbf{w}(\cdot, 0)\|^{2} \exp \left(2 \lambda_{\max } t\right)\right\}^{p / 2}
\end{aligned}
$$




$$
\begin{aligned}
\leq C_{3}\left\{\|\mathbf{w}(\cdot, 0)\|_{H^{2}}^{2}\right. & \\
& \left.+\|\mathbf{w}(\cdot, 0)\|^{2} \exp \left(2 \lambda_{\max } t\right)\right\}^{p / 2},
\end{aligned}
$$

where $C_{3}=\max \left\{\left[\left(E_{1}^{2}+1\right) \chi^{2}\right]^{p / 2},\left\{4 C_{1}^{2}\left[1+\left(E_{1}^{2}+1\right) C^{\prime} /\right.\right.\right.$ $\left.\left.\lambda_{\max }\right]^{p / 2}\right\}$.

(2) If $\chi^{2}<1$, from (70), we have

$$
\begin{aligned}
\chi^{2} \sum_{|\alpha|=2}\left\|D^{\alpha} \mathbf{w}(\cdot, t)\right\|^{2} \\
\leq \sum_{|\alpha|=2} \int_{\mathbb{T}^{d}}\left\{\left|D^{\alpha} u(\cdot, 0)\right|^{2}+\chi^{2}\left|D^{\alpha} v(\cdot, 0)\right|^{2}\right\} \mathbf{d x} \\
\quad+\frac{4 C_{1}^{2} C^{\prime}}{\lambda_{\max }}\|\mathbf{w}(\cdot, 0)\|^{2} \exp \left(2 \lambda_{\max } t\right) \\
\leq \sum_{|\alpha|=2}\left\|D^{\alpha} \mathbf{w}(\cdot, 0)\right\|^{2} \\
\quad+\frac{4 C_{1}^{2} C^{\prime}}{\lambda_{\max }}\|\mathbf{w}(\cdot, 0)\|^{2} \exp \left(2 \lambda_{\max } t\right) .
\end{aligned}
$$

Therefore,

$$
\begin{aligned}
\sum_{|\alpha|=2}\left\|D^{\alpha} \mathbf{w}(\cdot, t)\right\|^{2} & \\
\leq & \frac{1}{\chi^{2}} \sum_{|\alpha|=2}\left\|D^{\alpha} \mathbf{w}(\cdot, 0)\right\|^{2} \\
& +\frac{4 C_{1}^{2} C^{\prime}}{\chi^{2} \lambda_{\max }}\|\mathbf{w}(\cdot, 0)\|^{2} \exp \left(2 \lambda_{\max } t\right) .
\end{aligned}
$$

Combining (68) and (74), we have

$$
\begin{aligned}
&\|\mathbf{w}(\cdot, t)\|_{H^{2}}^{p} \leq\left\{\frac{E_{1}^{2}+1}{\chi^{2}} \sum_{|\alpha|=2}\left\|D^{\alpha} \mathbf{w}(\cdot, 0)\right\|^{2}\right. \\
&+4 C_{1}^{2}\left\{1+\frac{\left(E_{1}^{2}+1\right) C^{\prime}}{\lambda_{\max } \chi^{2}}\right\} \\
&\left.\times\|\mathbf{w}(\cdot, 0)\|^{2} \exp \left(2 \lambda_{\max } t\right)\right\}^{p / 2} \\
& \leq C_{3}\left\{\|\mathbf{w}(\cdot, 0)\|_{H^{2}}^{2}\right. \\
&\left.+\|\mathbf{w}(\cdot, 0)\|^{2} \exp \left(2 \lambda_{\max } t\right)\right\}^{p / 2},
\end{aligned}
$$

where $C_{3}=\max \left\{\left[\left(E_{1}^{2}+1\right) / \chi^{2}\right]^{p / 2},\left\{4 C_{1}^{2}\left[1+\left(E_{1}^{2}+1\right) C^{\prime} /\right.\right.\right.$ $\left.\left.\left.\lambda_{\max } \chi^{2}\right]\right\}^{p / 2}\right\}$.

\section{Main Result}

Let $\theta$ be a small fixed constant, and let $\lambda_{\max }$ be the dominant eigenvalue, which is the maximal growth rate. We also denote the gap between the largest growth rate $\lambda_{\max }$ and the rest by $v>0$. Then for $\delta>0$ arbitrary small, we define the escape time $T^{\delta}$ by

$$
\theta=\delta \exp \left(\lambda_{\max } T^{\delta}\right)
$$

or equivalently

$$
T^{\delta}=\frac{1}{\lambda_{\max }} \ln \frac{\theta}{\delta} .
$$

Our main result of this paper is the following theorem.

Theorem 6. Assume that the set of $q^{2}=\sum_{i=1}^{d} q_{i}^{2}$ satisfying instability criterion (16) is not empty for given parameters $\chi$ and $\beta$. Let

$$
\mathbf{w}_{0}(\mathbf{x})=\sum_{\mathbf{q} \in \Omega}\left\{w_{\mathbf{q}}^{-} \mathbf{r}_{-}(\mathbf{q})+w_{\mathbf{q}}^{+} \mathbf{r}_{+}(\mathbf{q})\right\} e_{\mathbf{q}}(\mathbf{x}) \in H^{2},
$$

such that $\left\|\mathbf{w}_{0}\right\|=1$. Then there exist constants $\delta_{0}>0, C>0$, and $\theta>0$, depending on $\chi$ and $\beta$, such that for all $0<\delta \leq \delta_{0}$, if the initial perturbation of the steady state $[\bar{U}, \bar{V}]$ is $\mathbf{w}^{\delta}(\cdot, 0)=$ $\delta \mathbf{w}_{0}$, then its nonlinear evolution $\mathbf{w}^{\delta}(\cdot, t)$ satisfies

$$
\begin{array}{r}
\left\|\mathbf{w}^{\delta}(\cdot, t)-\delta e^{\lambda_{\max } t} \sum_{\mathbf{q} \in \Omega_{\max }} w_{\mathbf{q}}^{+} \mathbf{r}_{+}(\mathbf{q}) e_{\mathbf{q}}(\mathbf{x})\right\| \\
\leq C\left\{e^{-\gamma t}+\delta\left\|\mathbf{w}_{0}\right\|_{H^{2}}^{2}+\delta^{p}\left\|\mathbf{w}_{0}\right\|_{H^{2}}^{p+1}\right. \\
\left.+\delta e^{\lambda_{\max } t}+\delta^{p} e^{p \lambda_{\max } t}\right\} \delta e^{\lambda_{\max } t},
\end{array}
$$

for $0 \leq t \leq T^{\delta}$, and $v>0$ is the gap between $\lambda_{\max }$ and the rest of $\lambda_{\mathbf{q}}$ in (15).

Proof. Let $\mathbf{w}^{\delta}(\mathbf{x}, t)$ be the solutions of (10) with initial data $\mathbf{w}^{\delta}(\cdot, 0)=\delta \mathbf{w}_{0}$. We define

$$
\begin{gathered}
T^{*}=\sup \left\{t \mid\left\|\mathbf{w}^{\delta}(\cdot, t)-\delta e^{\mathfrak{Q} t} \mathbf{w}_{0}\right\| \leq \frac{C_{1}}{2} \delta \exp \left(\lambda_{\max } t\right)\right\}, \\
T^{* *}=\sup \left\{t \mid\left\|\mathbf{w}^{\delta}(\cdot, t)\right\|_{H^{2}}+\left\|\mathbf{w}^{\delta}(\cdot, t)\right\|_{H^{2}}^{p}\right. \\
\leq \frac{1}{C_{0}^{*}} \min \left\{\frac{1}{4\left(\chi+2^{p-1}(p+1)^{2}\right)},\right. \\
\left.\left.\frac{\chi^{2}}{2\left(\chi+2^{p-1}(p+1)^{2}\right)}\right\}\right\} .
\end{gathered}
$$

Choose $\theta$, such that

$$
\begin{gathered}
C_{0}^{*} C_{3}\left\{\theta+\frac{4^{p}}{p} C_{3}^{(p-1) / 2} \theta^{p}\right\} \\
<\min \left\{\frac{\lambda_{\max }}{4(\chi+1)}, \frac{1}{8\left(\chi+2^{p-1}(p+1)^{2}\right)},\right. \\
\left.\frac{\chi^{2}}{4\left(\chi+2^{p-1}(p+1)^{2}\right)}\right\} .
\end{gathered}
$$


Now, we establish estimates for $H^{2}$ norm of $\mathbf{w}^{\delta}(\mathbf{x}, t)$ for $t \leq \min \left\{T^{\delta}, T^{*}, T^{* *}\right\}$. For $0<t \leq T^{*}$, by Lemma 2 , we have

$$
\begin{aligned}
\left\|\mathbf{w}^{\delta}(\cdot, t)\right\| \leq & \left\|\delta e^{\mathfrak{Q t}} \mathbf{w}_{0}\right\|+\frac{C_{1}}{2} \delta \exp \left(\lambda_{\max } t\right) \\
\leq & C_{1} \delta \exp \left(\lambda_{\max } t\right)\|\mathbf{w}(\cdot, 0)\| \\
& +\frac{C_{1}}{2} \delta \exp \left(\lambda_{\max } t\right) \\
= & \frac{3}{2} C_{1} \delta \exp \left(\lambda_{\max } t\right) .
\end{aligned}
$$

Applying Lemma 5 and the bootstrap argument yields

$$
\left\|\mathbf{w}^{\delta}(\cdot, t)\right\|_{H^{2}} \leq \sqrt{C_{3}}\left\{\delta\left\|\mathbf{w}_{0}\right\|_{H^{2}}+\delta \exp \left(\lambda_{\max } t\right)\right\} .
$$

From this and $(a+b)^{p} \leq 2^{p-1}\left(a^{p}+b^{p}\right)(a \geq 0, b \geq 0, p \geq 1)$, we can obtain

$$
\begin{aligned}
& \left\|\mathbf{w}^{\delta}(\cdot, t)\right\|_{H^{2}}^{p+1} \\
& \quad \leq 2^{p} C_{3}^{(p+1) / 2}\left\{\delta^{p+1}\left\|\mathbf{w}_{0}\right\|_{H^{2}}^{p+1}+\delta^{p+1} \exp (p+1) \lambda_{\max } t\right\} .
\end{aligned}
$$

Applying Duhamel's principle, we know that the solution of $(10)$ is

$$
\begin{aligned}
& \mathbf{w}^{\delta}(\cdot, t)=\delta e^{\mathfrak{R} t} \mathbf{w}_{0} \\
& -\int_{0}^{t} e^{\mathfrak{Q}(t-\tau)}\left[\chi \nabla\left(u^{\delta} \nabla v^{\delta}\right)\right. \\
& \left.+u^{\delta}\left(u^{\delta}+1\right)^{p}-u^{\delta}, 0\right] d \tau .
\end{aligned}
$$

Using Lemma 2, (56), (58), and Lemma 5 yields, for $0 \leq t \leq$ $\min \left\{T^{\delta}, T^{*}, T^{* *}\right\}$,

$$
\begin{gathered}
\left\|\mathbf{w}^{\delta}(\cdot, t)-\delta e^{\Omega t} \mathbf{w}_{0}\right\| \\
\leq C_{1} \int_{0}^{t} e^{\lambda_{\max }(t-\tau)} \| u^{\delta}(\tau)-u^{\delta}(\tau)\left(u^{\delta}(\tau)+1\right)^{p} \\
-\chi \nabla\left(u^{\delta}(\tau) \nabla v^{\delta}(\tau)\right) \| d \tau \\
\leq C_{1} M_{3} \int_{0}^{t} e^{\lambda_{\max }(t-\tau)}\left\|\mathbf{w}^{\delta}(\tau)\right\|_{H^{2}}^{p+1} d \tau \\
+C_{1}\left(E_{1}^{2} \chi+E_{0} \chi\right) \int_{0}^{t} e^{\lambda_{\max }(t-\tau)}\left\|\mathbf{w}^{\delta}(\tau)\right\|_{H^{2}}^{2} d \tau \\
\leq C_{1} C_{0}^{*}\{\chi+1\} \int_{0}^{t} e^{\lambda_{\max }(t-\tau)}\left\{\left\|\mathbf{w}^{\delta}(\tau)\right\|_{H^{2}}^{2}\right. \\
\left.+\left\|\mathbf{w}^{\delta}(\tau)\right\|_{H^{2}}^{p+1}\right\} d \tau,
\end{gathered}
$$

where $C_{0}^{*}=\max \left\{M_{3}, E_{1}^{2}+E_{0}\right\}$. Notice that $t \leq$ $\min \left\{T^{\delta}, T^{*}, T^{* *}\right\}$. Thus

$$
\begin{aligned}
\| \mathbf{w}^{\delta}(t) & -\delta e^{\Omega t} \mathbf{w}_{0} \| \\
\leq & C_{1} C_{0}^{*} C_{3}(\chi+1) \\
& \times \int_{0}^{t} e^{\lambda_{\max }(t-\tau)}\left\{\delta^{2}\left\|\mathbf{w}_{0}\right\|_{H^{2}}^{2}+\delta^{2} e^{2 \lambda_{\max } \tau}\right\} d \tau \\
& +2^{p} C_{1} C_{0}^{*} C_{3}^{(p+1) / 2}(\chi+1) \\
& \times \int_{0}^{t} e^{\lambda_{\max }(t-\tau)}\left\{\delta^{p+1}\left\|\mathbf{w}_{0}\right\|_{H^{2}}^{p+1}+\delta^{p+1} e^{(p+1) \lambda_{\max } \tau}\right\} d \tau \\
\leq & C_{1} C_{0}^{*} C_{3}(\chi+1) \\
& \times\left\{\frac{\delta\left\|\mathbf{w}_{0}\right\|_{H^{2}}^{2}+2^{p} C_{3}^{(p-1) / 2} \delta^{p}\left\|\mathbf{w}_{0}\right\|_{H^{2}}^{p+1}}{\lambda_{\max }}\right. \\
& \left.\quad+\frac{\delta e^{\lambda_{\max } t}+\left(2^{p} / p\right) C_{3}^{(p-1) / 2} \delta^{p} e^{p \lambda_{\max } t}}{\lambda_{\max }}\right\} \delta e^{\lambda_{\max } t} .
\end{aligned}
$$

For $\delta>0$ sufficiently small, we claim that

$$
T^{\delta}=\min \left\{T^{\delta}, T^{*}, T^{* *}\right\} .
$$

If $T^{* *}$ is the smallest, we can let $t=T^{* *} \leq T^{\delta}$ in (84) and (85) to obtain

$$
\begin{aligned}
&\left\|\mathbf{w}^{\delta}\left(\cdot, T^{* *}\right)\right\|_{H^{2}}+\left\|\mathbf{w}\left(\cdot, T^{* *}\right)\right\|_{H^{2}}^{p} \leq{ }^{p-1} C_{3}^{p / 2} \delta^{p}\left\|\mathbf{w}_{0}\right\|_{H^{2}}^{p}+\sqrt{C_{3}} \delta\left\|\mathbf{w}_{0}\right\|_{H^{2}} \\
&+C_{3}^{1 / 2}\left(\theta+2^{p-1} C_{3}^{(p-1) / 2} \theta^{p}\right) \\
&< \frac{1}{C_{0}^{*}} \min \left\{\frac{1}{4\left(\chi+2^{p-1}(p+1)^{2}\right)},\right. \\
&\left.\frac{\chi^{2}}{2\left(\chi+2^{p-1}(p+1)^{2}\right)}\right\},
\end{aligned}
$$

for $2^{p-1} C_{3}^{p / 2} \delta^{p}\left\|\mathbf{w}_{0}\right\|_{H^{2}}^{p}+\sqrt{C_{3}} \delta\left\|\mathbf{w}_{0}\right\|_{H^{2}} \leq\left(1 / 2 C_{0}^{*}\right) \min \{1 / 4(\chi+$ $\left.\left.2^{p-1}(p+1)^{2}\right), \chi^{2} / 2\left(\chi+2^{p-1}(p+1)^{2}\right)\right\}$ for $\delta$ small, by our choice of $\theta$ in (82) with $C_{3} \geq 1$. This is a contradiction to the definition of $T^{* *}$. On the other hand, if $T^{*}$ is the smallest, we let $t=T^{*}$ in (88) to get

$$
\begin{aligned}
& \left\|\mathbf{w}^{\delta}\left(\cdot, T^{*}\right)-\delta e^{R T^{*}} \mathbf{w}_{0}\right\| \\
& \leq C_{1} C_{0}^{*} C_{3}(\chi+1) \\
& \quad \times\left\{\frac{\delta\left\|\mathbf{w}_{0}\right\|_{H^{2}}^{2}+2^{p} C_{3}^{(p-1) / 2} \delta^{p}\left\|\mathbf{w}_{0}\right\|_{H^{2}}^{p+1}}{\lambda_{\max }}\right. \\
& \left.+\frac{\theta+\left(2^{p} / p\right) C_{3}^{(p-1) / 2} \theta^{p}}{\lambda_{\max }}\right\} \delta e^{\lambda_{\max } T^{*}} \\
& <\frac{C_{1}}{2} \delta e^{\lambda_{\max } T^{*}},
\end{aligned}
$$


for $C_{0}^{*} C_{3}(\chi+1)\left(\delta\left\|\mathbf{w}_{0}\right\|_{H^{2}}^{2}+2^{p} C_{3}^{(p-1) / 2} \delta^{p}\left\|\mathbf{w}_{0}\right\|_{H^{2}}^{p+1}\right) / \lambda_{\max }<1 / 4$ for $\delta$ small, by our choice of $\theta$ in (82). This again contradicts the definition of $T^{*}$. Hence, the desired assertion follows.

From (24), we obtain

$$
\begin{aligned}
& \left\|\mathbf{w}^{\delta}(\cdot, t)-\delta e^{\Omega t} \mathbf{w}_{0}\right\| \\
& \geq\left\|\mathbf{w}^{\delta}(\cdot, t)-\delta e^{\lambda_{\max } t} \sum_{\mathbf{q} \in \Omega_{\max }} w_{\mathbf{q}}^{+} \mathbf{r}_{+}(\mathbf{q}) e_{\mathbf{q}}(\mathbf{x})\right\| \\
& -\left\|\delta \sum_{\mathbf{q} \in \Omega_{\max }} w_{\mathbf{q}}^{-} \mathbf{r}_{-}(\mathbf{q}) \exp \left(\lambda_{\mathbf{q}}^{-} t\right) e_{\mathbf{q}}(\mathbf{x})\right\| \\
& -\| \delta \sum_{\mathbf{q} \in \Omega \backslash \Omega_{\max }}\left\{w_{\mathbf{q}}^{-} \mathbf{r}_{-}(\mathbf{q}) \exp \left(\lambda_{\mathbf{q}}^{-} t\right)\right. \\
& =\left\|\mathbf{w}^{\delta}(\cdot, t)-\delta e^{\lambda_{\max } t} \sum_{\mathbf{q} \in \Omega_{\max }} w_{\mathbf{q}}^{+} \mathbf{r}_{+}(\mathbf{q}) e_{\mathbf{q}}(\mathbf{x})\right\|-I_{1}-I_{2},
\end{aligned}
$$

that is,

$$
\begin{gathered}
\left\|\mathbf{w}^{\delta}(\cdot, t)-\delta e^{\lambda_{\max } t} \sum_{\mathbf{q} \in \Omega_{\max }} w_{\mathbf{q}}^{+} \mathbf{r}_{+}(\mathbf{q}) e_{\mathbf{q}}(\mathbf{x})\right\| \\
\leq\left\|\mathbf{w}^{\delta}(\cdot, t)-\delta e^{\mathfrak{Q} t} \mathbf{w}_{0}\right\|+I_{1}+I_{2} .
\end{gathered}
$$

Using (34), we deduce that

$$
\begin{aligned}
I_{1}^{2} & =\left\|\delta \sum_{\mathbf{q} \in \Omega_{\max }} w_{\mathbf{q}}^{-} \mathbf{r}_{-}(\mathbf{q}) \exp \left(\lambda_{\mathbf{q}}^{-} t\right) e_{\mathbf{q}}(\mathbf{x})\right\|^{2} \\
& \leq 4 \delta^{2} e^{2\left(\lambda_{\max }-\gamma\right) t}\left(\frac{\pi}{2}\right)^{d} \sum_{\mathbf{q} \in \Omega_{\max }} E_{2}^{2} E_{3}^{2} q^{6}\left|\mathbf{w}_{q}\right|^{2} .
\end{aligned}
$$

From (18) we know that there is one (or two) $q^{2}$ satisfying $\lambda^{+}\left(q^{2}\right)=\lambda_{\max }$. If there is only one $q^{2}$ satisfying $\lambda^{+}\left(q^{2}\right)=$ $\lambda_{\max }$, we denote it by $q_{\max }^{2}$ and if there are $q_{1}^{2}$ and $q_{2}^{2}$ satisfying $\lambda^{+}\left(q^{2}\right)=\lambda_{\max }$, we can let $q_{\max }^{2}=\max \left\{q_{1}^{2}, q_{2}^{2}\right\}$. From (94), we obtain

$$
\begin{aligned}
I_{1}^{2} & \leq 4 \delta^{2} e^{2\left(\lambda_{\max }-\gamma\right) t} E_{2}^{2} E_{3}^{2} q_{\max }^{6}\left(\frac{\pi}{2}\right)^{d} \sum_{\mathbf{q} \in \Omega_{\max }}\left|\mathbf{w}_{\mathbf{q}}\right|^{2} \\
& \leq 4 E_{2}^{2} E_{3}^{2} q_{\max }^{6} \delta^{2} e^{2\left(\lambda_{\max }-v\right) t}\left\|\mathbf{w}_{0}\right\|^{2},
\end{aligned}
$$

that is,

$$
I_{1} \leq 2 E_{2} E_{3} q_{\max }^{3} \delta e^{\left(\lambda_{\max }-v\right) t}=C^{*} \delta e^{\left(\lambda_{\max }-v\right) t}
$$

where $C^{*}=2 E_{2} E_{3} q_{\max }^{3}$. Now, we consider $I_{2}$

$$
\begin{aligned}
I_{2}^{2}=\delta^{2}\left(\frac{\pi}{2}\right)^{d} \sum_{\mathbf{q} \in \Omega \backslash \Omega_{\max }} & \left\{\exp \left(2 \lambda_{\mathbf{q}}^{+} t\right) w_{\mathbf{q}}^{+2}\left|\mathbf{r}_{+}(\mathbf{q})\right|^{2}\right. \\
& +2 \exp \left(\left(\lambda_{\mathbf{q}}^{+}+\lambda_{\mathbf{q}}^{-}\right) t\right) \\
& \times w_{\mathbf{q}}^{+} w_{\mathbf{q}}^{-}\left(\mathbf{r}_{+}(\mathbf{q}), \mathbf{r}_{-}(\mathbf{q})\right) \\
& \left.+\exp \left(2 \lambda_{\mathbf{q}}^{-} t\right) w_{\mathbf{q}}^{-2}\left|\mathbf{r}_{-}(\mathbf{q})\right|^{2}\right\} .
\end{aligned}
$$

From (39) and (41), we get

$$
\begin{aligned}
& I_{2}^{2} \leq \delta^{2} e^{2\left(\lambda_{\max }-v\right) t}\left(\frac{\pi}{2}\right)^{d} \\
& \times \sum_{\mathbf{q} \in \Omega \backslash \Omega_{\max }}\left\{w_{\mathbf{q}}^{+2}\left|\mathbf{r}_{+}(\mathbf{q})\right|^{2}\right. \\
&+2 w_{\mathbf{q}}^{+} w_{\mathbf{q}}^{-}\left(\mathbf{r}_{+}(\mathbf{q}), \mathbf{r}_{-}(\mathbf{q})\right) \\
&\left.+w_{\mathbf{q}}^{-2}\left|\mathbf{r}_{-}(\mathbf{q})\right|^{2}\right\} \\
& \leq \delta^{2} e^{2\left(\lambda_{\max }-v\right) t}\left(\frac{\pi}{2}\right)^{d} \sum_{\mathbf{q} \in \Omega}\left|\mathbf{w}_{\mathbf{q}}\right|^{2} \\
&=\delta^{2} e^{2\left(\lambda_{\max }-v\right) t},
\end{aligned}
$$

that is,

$$
I_{2} \leq \delta e^{\left(\lambda_{\max }-v\right) t} .
$$

From (88), (96), and (99), one can obtain

$$
\begin{aligned}
& \left\|\mathbf{w}^{\delta}(\cdot, t)-\delta e^{\lambda_{\max } t} \sum_{\mathbf{q} \in \Omega_{\max }} w_{\mathbf{q}}^{+} \mathbf{r}_{+}(\mathbf{q}) e_{\mathbf{q}}(\mathbf{x})\right\| \\
& \leq C_{1} C_{0}^{*} C_{3}(\chi+1) \\
& \times\left\{\frac{\delta\left\|\mathbf{w}_{0}\right\|_{H^{2}}^{2}+2^{p} C_{3}^{(p-1) / 2} \delta^{p}\left\|\mathbf{w}_{0}\right\|_{H^{2}}^{p+1}}{\lambda_{\max }}\right. \\
& \left.+\frac{\delta e^{\lambda_{\max } t}+\left(2^{p} / p\right) C_{3}^{(p-1) / 2} \delta^{p} e^{p \lambda_{\max } t}}{\lambda_{\max }}\right\} \delta e^{\lambda_{\max } t} \\
& +C^{*} \delta e^{\left(\lambda_{\max }-v\right) t}+\delta e^{\left(\lambda_{\max }-v\right) t} \\
& \leq\left\{\left(C^{*}+1\right) e^{-\nu t}+\frac{\left(4^{p} / p\right) C_{1} C_{0}^{*} C_{3}^{p+1}}{\lambda_{\max }}(\chi+1)\right. \\
& \times\left\{\delta\left\|\mathbf{w}_{0}\right\|_{H^{2}}^{2}+\delta^{p}\left\|\mathbf{w}_{0}\right\|_{H^{2}}^{p+1}\right. \\
& \left.\left.+\delta e^{\lambda_{\max } t}+\delta^{p} e^{p \lambda_{\max } t}\right\}\right\} \delta e^{\lambda_{\max } t} \\
& \leq C\left\{e^{-v t}+\delta\left\|\mathbf{w}_{0}\right\|_{H^{2}}^{2}+\delta^{p}\left\|\mathbf{w}_{0}\right\|_{H^{2}}^{p+1}\right. \\
& \left.+\delta e^{\lambda_{\max } t}+\delta^{p} e^{p \lambda_{\max } t}\right\} \delta e^{\lambda_{\max } t},
\end{aligned}
$$


where $C=\max \left\{C^{*}+1,\left(\left(4^{p} / p\right) C_{1} C_{0}^{*} C_{3}^{p+1} / \lambda_{\max }\right)(\chi+1)\right\}$. This completes the proof.

\section{Conclusion}

Notice that for $0 \leq t \leq T^{\delta}, \delta e^{\lambda_{\max } t} \leq \theta$ is sufficiently small. As long as $w_{\mathbf{q}_{0}}^{+} \neq 0$ for at least one $\mathbf{q}_{0} \in \Omega_{\text {max }}$, which is generic for perturbations, the corresponding fastest growing modes

$$
\begin{aligned}
\left\|\delta e^{\lambda_{\max } t} \sum_{\mathbf{q} \in \Omega_{\max }} w_{\mathbf{q}}^{+} \mathbf{r}_{+}(\mathbf{q}) e_{\mathbf{q}}(\mathbf{x})\right\| \\
=\delta e^{\lambda_{\max } t}\left(\frac{\pi}{2}\right)^{d / 2} \\
\times\left(\sum_{\mathbf{q} \in \Omega_{\max }}\left|w_{\mathbf{q}}^{+}\right|^{2}\left|\mathbf{r}_{+}(\mathbf{q})\right|^{2}\right)^{1 / 2} \\
\geq \delta e^{\lambda_{\max } t}\left|w_{\mathbf{q}_{0}}^{+}\right|\left|\mathbf{r}_{+}\left(\mathbf{q}_{0}\right)\right|,
\end{aligned}
$$

have the dominant leading order of $\delta e^{\lambda_{\max } t}$. Theorem 6 implies that the dynamics of a general perturbation is characterized by such linear dynamics over a long time period of $\varepsilon T^{\delta} \leq t \leq T^{\delta}$, for any $\varepsilon>0$ (see [10]). In particular, choose a fixed $\mathbf{q}_{0}=\left(q_{01}, q_{02}, \ldots, q_{0 d}\right) \in \Omega_{\max }$ and let

$$
\mathbf{w}_{0}(\mathbf{x})=\frac{\mathbf{r}_{+}\left(\mathbf{q}_{0}\right)}{\left|\mathbf{r}_{+}\left(\mathbf{q}_{0}\right)\right|} e_{\mathbf{q}_{0}}(\mathbf{x}) .
$$

Then

$$
\left\|\mathbf{w}_{0}(\mathbf{x})\right\|_{H^{2}}=\left\{\left(\frac{\pi}{2}\right)^{d}\left(1+\left|\mathbf{q}_{0}\right|^{2}+\left|\mathbf{q}_{0}\right|^{4}\right)\right\}^{1 / 2} .
$$

Therefore, if $t \leq T^{\delta}$, we can obtain from (100)

$$
\begin{aligned}
& \| \mathbf{w}^{\delta}(\cdot, t)-\delta e^{\lambda_{\max } T^{\delta}} \frac{\mathbf{r}_{+}\left(\mathbf{q}_{0}\right)}{\left|\mathbf{r}_{+}\left(\mathbf{q}_{0}\right)\right|} e_{\mathbf{q}_{0}}(\mathbf{x}) \| \\
& \leq C\left\{e^{-\nu T^{\delta}}+\delta\left\|\mathbf{w}_{0}\right\|_{H^{2}}^{2}+\delta^{p}\left\|\mathbf{w}_{0}\right\|_{H^{2}}^{p+1}\right. \\
&\left.+\delta e^{\lambda_{\max } T^{\delta}}+\delta^{p} e^{p \lambda_{\max } T^{\delta}}\right\} \delta e^{\lambda_{\max } T^{\delta}} \\
& \leq C\left\{\theta \exp \left(\frac{-v}{\lambda_{\max }} \ln \frac{\theta}{\delta}\right)\right. \\
&+\theta \delta\left(\frac{\pi}{2}\right)^{d}\left(1+\left|\mathbf{q}_{0}\right|^{2}+\left|\mathbf{q}_{0}\right|^{4}\right) \\
&+\theta \delta^{p}\left(\frac{\pi}{2}\right)^{(p+1) d / 2} \\
& \times\left(1+\left|\mathbf{q}_{0}\right|^{2}+\left|\mathbf{q}_{0}\right|^{4}\right)^{(p+1) / 2} \\
&\left.+\theta^{2}+\theta^{p+1}\right\} .
\end{aligned}
$$

Note that $\delta \leq \theta, \theta$, and $\nu$ are fixed constants, and $\mathbf{q}_{0}$ is a fixed vector. Hence, we have

$$
\begin{gathered}
\left\|\mathbf{w}^{\delta}(\cdot, t)-\delta e^{\lambda_{\max } T^{\delta}} \frac{\mathbf{r}_{+}\left(\mathbf{q}_{0}\right)}{\left|\mathbf{r}_{+}\left(\mathbf{q}_{0}\right)\right|} e^{\mathbf{q}_{0}}(\mathbf{x})\right\| \\
\leq C C_{4}\left\{\delta^{\nu / \lambda_{\max }}+\theta^{2}+\theta^{p+1}\right\},
\end{gathered}
$$

where $C_{4}=\max \left\{\theta^{1-\nu / \lambda_{\max }},(\pi / 2)^{(p+1) d / 2}\left(1+\left|\mathbf{q}_{0}\right|^{2}+\left|\mathbf{q}_{0}\right|^{4}\right)^{(p+1) / 2}+\right.$ $1\}$. Thus,

$$
\begin{aligned}
\left\|\mathbf{w}^{\delta}(\cdot, t)\right\| \geq & \left\|\delta e^{\lambda_{\max } T^{\delta}} \frac{\mathbf{r}_{+}\left(\mathbf{q}_{0}\right)}{\left|\mathbf{r}_{+}\left(\mathbf{q}_{0}\right)\right|} e_{\mathbf{q}_{0}}(\mathbf{x})\right\| \\
& -C C_{4}\left\{\delta^{\nu / \lambda_{\max }}+\theta^{2}+\theta^{p+1}\right\} \\
\geq & \theta-C^{* *}\left\{\delta^{\nu / \lambda_{\max }}+\theta^{2}+\theta^{p+1}\right\} .
\end{aligned}
$$

Let $\theta$ be sufficiently small so that $\theta / 2 C^{* *}-\theta^{2}-\theta^{p+1}>0$ holds. Then $\delta_{1}=:\left(\theta / 2 C^{* *}-\theta^{2}-\theta^{p+1}\right)^{\lambda_{\max } / \nu}$, and

$$
\begin{aligned}
\left\|\mathbf{w}^{\delta}(\cdot, t)\right\| & \geq \theta-C^{* *}\left\{\delta^{\nu / \lambda_{\max }}+\theta^{2}+\theta^{p+1}\right\} \\
& \geq \frac{\theta}{2}>0, \quad 0 \leq \delta \leq \delta_{1} .
\end{aligned}
$$

This implies nonlinear instability as $\delta \rightarrow 0$. Then instability occurs before the possible blow-up time.

Recall that the unique positive equilibrium point of (2) without chemotaxis is locally asymptotically stable. This means that the instability in (2) is called chemotaxis-driven instability since the presence of chemotaxis is essential for the instability mechanism leading to nonuniform patterns in the system (2).

\section{Acknowledgments}

The authors would like to thank the referee for their helpful comments. This work is supported by the China National Natural Science Foundation (no. 11061031; 11261053) and the Fundamental Research Funds for the Gansu University.

\section{References}

[1] M. Mimura and T. Tsujikawa, "Aggregating pattern dynamics in a chemotaxis model including growth," Physica A, vol. 230, no. 3-4, pp. 499-543, 1996.

[2] J. I. Tello and M. Winkler, "A chemotaxis system with logistic source," Communications in Partial Differential Equations, vol. 32, no. 6, pp. 849-877, 2007.

[3] N. Kurata, K. Kuto, K. Osaki, T. Tsujikawa, and T. Sakurai, "Bifurcation phenomena of pattern solution to Mimura-Tsujikawa model in one dimension," Gakuto International Series. Mathematical Sciences and Applications, vol. 29, pp. 265-278, 2008.

[4] K. Kuto, K. Osaki, T. Sakurai, and T. Tsujikawa, "Spatial pattern formation in a chemotaxis-diffusion-growth model," Physica D, vol. 241, no. 19, pp. 1629-1639, 2012.

[5] S. Banerjee, A. P. Misra, and L. Rondoni, "Spatiotemporal evolution in a $(2+1)$-dimensional chemotaxis model," Physica A, vol. 391, no. 1-2, pp. 107-112, 2012. 
[6] K. J. Painter and T. Hillen, "Spatio-temporal chaos in a chemotaxis model," Physica D, vol. 240, no. 4-5, pp. 363-375, 2011.

[7] M. Aida, T. Tsujikawa, M. Efendiev, A. Yagi, and M. Mimura, "Lower estimate of the attractor dimension for a chemotaxis growth system," Journal of the London Mathematical Society, vol. 74, no. 2, pp. 453-474, 2006.

[8] M. Efendiev, E. Nakaguchi, and W. L. Wendland, "Uniform estimate of dimension of the global attractor for a semi-discretized chemotaxis-growth system," Discrete and Continuous Dynamical Systems, Supplement, pp. 334-343, 2007.

[9] E. Nakaguchi and M. Efendiev, "On a new dimension estimate of the global attractor for chemotaxis-growth systems," Osaka Journal of Mathematics, vol. 45, no. 2, pp. 273-281, 2008.

[10] Y. Guo and H. J. Hwang, "Pattern formation (I): the Keller-Segel model," Journal of Differential Equations, vol. 249, no. 7, pp. 15191530, 2010.

[11] E. F. Keller and L. A. Segel, "Initiation of slime mold aggregation viewed as an instability," Journal of Theoretical Biology, vol. 26, no. 3, pp. 399-415, 1970.

[12] D. Henry, Geometric Theory of Semilinear Parabolic Equations, Springer, New York, NY, USA, 1993.

[13] O. A. Ladyzenskaja, V. A. Solonnikov, and N. N. Uralceva, Linear and Quasilinear Partial Di Erential, Equations of Parabolic Type, vol. 23 of Translations of Mathematical Monographs, American Mathematical Society, 1968. 


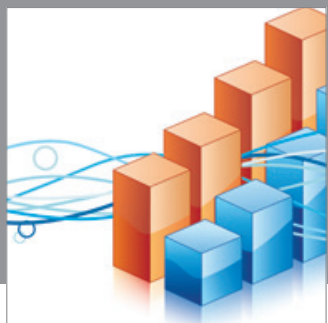

Advances in

Operations Research

mansans

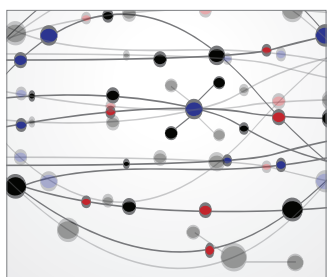

The Scientific World Journal
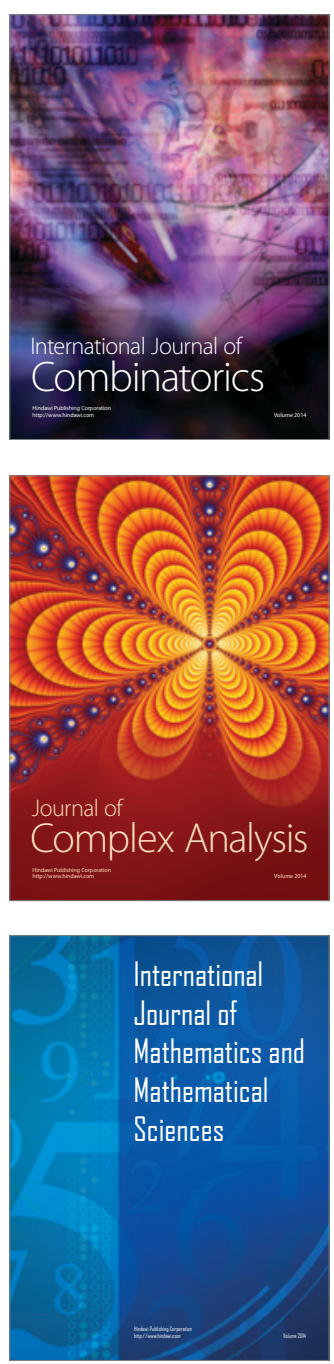
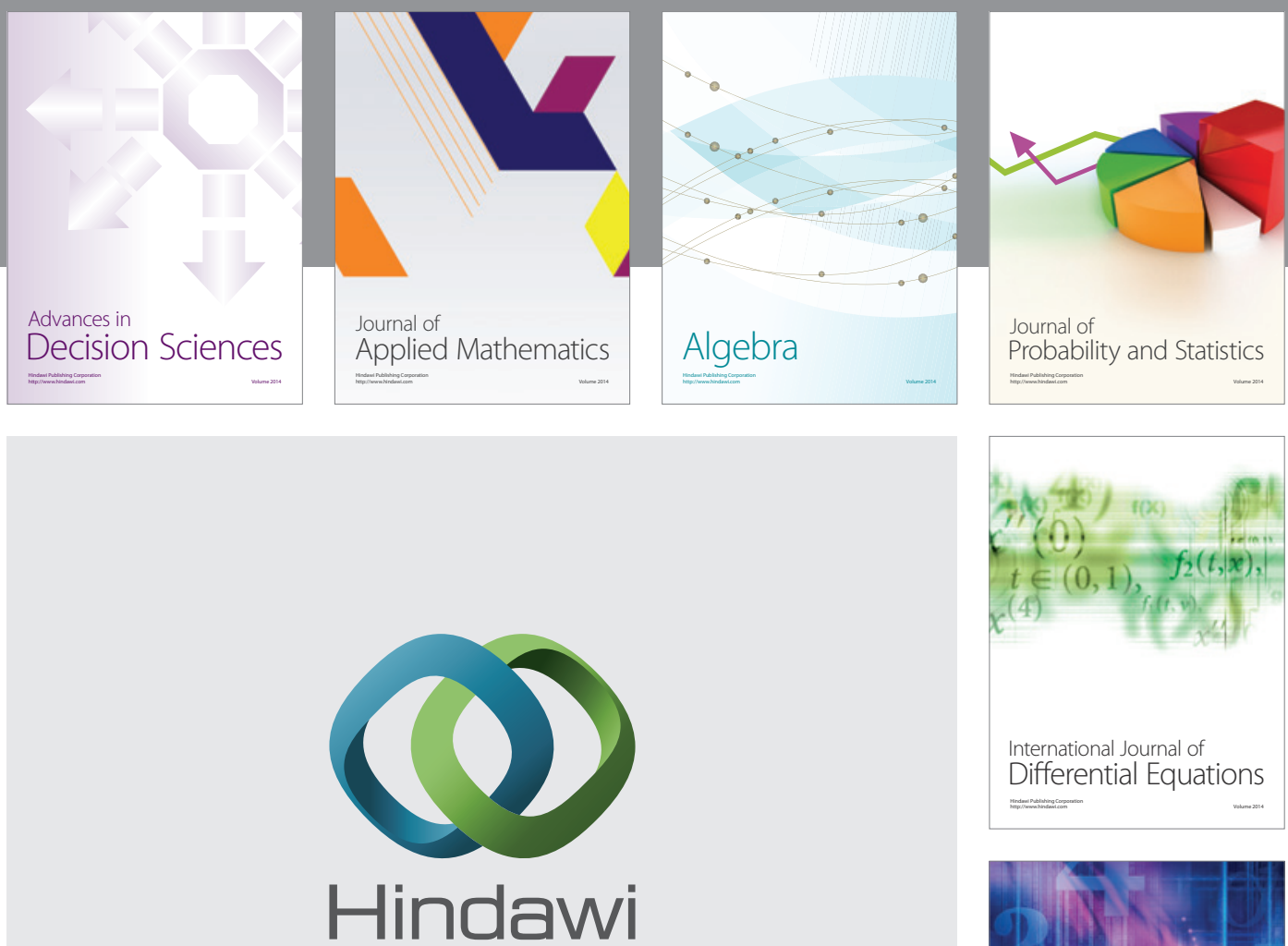

Submit your manuscripts at http://www.hindawi.com
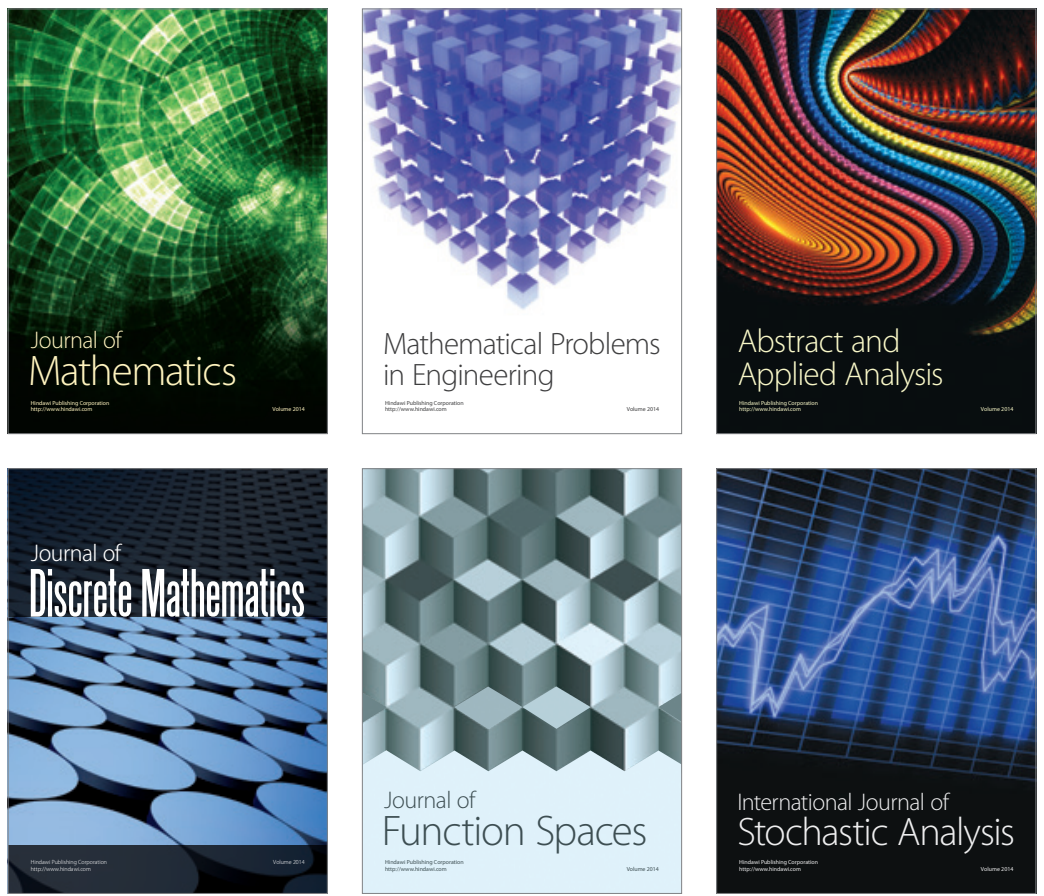

Journal of

Function Spaces

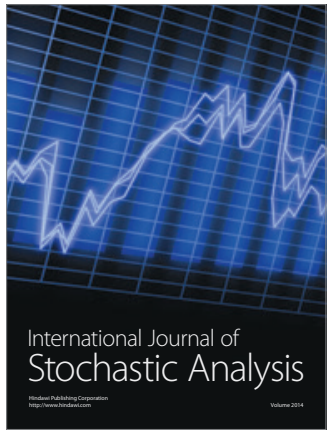

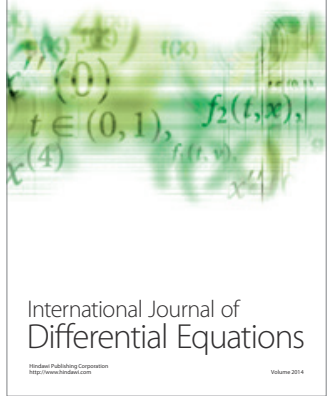
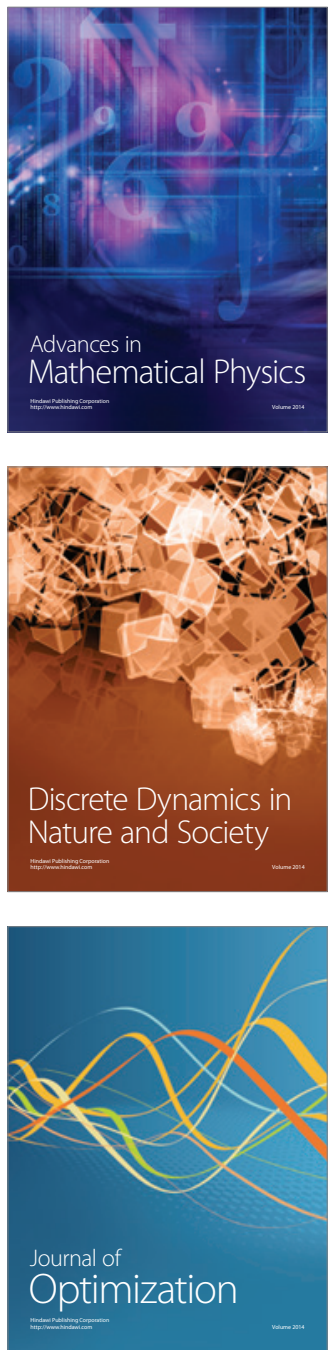